\title{
TakoTsubo cardiomyopathy: unravelling the malignant consequences of a benign disease with cardiac magnetic resonance
}

\author{
Amardeep Ghosh Dastidar $^{1} \cdot$ Antonio Frontera $^{1} \cdot$ Alberto Palazzuoli $^{1}$ • \\ Chiara Bucciarelli-Ducci ${ }^{1}$
}

Published online: 21 April 2015

(c) Springer Science+Business Media New York 2015

\begin{abstract}
TakoTsubo cardiomyopathy (TCM) is a unique type of reversible cardiomyopathy that is precipitated by a stressful emotional or physical event. The increasing incidence is due to the greater use of emergency coronary angiography, newer cardiac biomarkers together with more sensitive cardiac imaging techniques. Few case reports have documented how TCM can present with malignant arrhythmias such as torsades de pointes caused by the repolarisation abnormalities or QTc prolongation. Although TCM is usually considered a benign reversible condition, its associated arrhythmic risk is increasingly recognised. TCM often presents as an acute coronary syndrome with unobstructed coronary arteries at angiography. In this patient population, cardiac magnetic resonance (CMR) is a useful tool to establish a differential diagnosis, discriminating TCM from acute myocarditis and myocardial infarction with spontaneous recanalisation. CMR is becoming a promising new diagnostic modality in risk stratifying patients with potential higher arrhythmic risk.
\end{abstract}

Keywords TakoTsubo cardiomyopathy - Cardiac MRI . CMR

Amardeep Ghosh Dastidar

a.ghoshdastidar@bristol.ac.uk

$\bowtie$ Chiara Bucciarelli-Ducci

c.bucciarelli-ducci@bristol.ac.uk

1 NIHR Cardiovascular Biomedical Research Unit, Bristol Heart Institute, University of Bristol, Bristol BS2 8HW, UK

\section{Introduction}

TakoTsubo cardiomyopathy (TCM) is a unique type of reversible cardiomyopathy that is precipitated by a stressful emotional or physical event. It is estimated that approximately $1-2 \%$ of all patients presenting as acute coronary syndrome have TCM [1]. The increasing incidence is due to the greater use of emergency coronary angiography and advanced cardiac imaging (CMR) and of newer more sensitive cardiac biomarkers. This is a fairly new clinical entity with initial reported cases from Japan in 1990, and the first report emerging from the USA in 1998 [1].

Whilst the diagnostic criteria of TCM have been validated [2], the underlying pathophysiological processes still remain unclear. Major psychological stress seems to play a key role in initiating a chain of biological events that cause temporary reductions in myocardial contractility. Catecholamine-mediated myocardial stunning is currently the most favoured hypothesis. It has been shown that patients with this condition have higher concentrations of circulating catecholamines than those with atherosclerotic acute myocardial infarction (MI) [3]. Relative oestrogen deficiency has been investigated as another cause, and interestingly, the condition is more often seen in postmenopausal women. Other proposed mechanisms include ischaemia-mediated stunning due to multi-vessel epicardial or microvascular spasm and myocarditis.

Although the diagnostic criteria are well established, knowledge of the more dangerous implications of TCM still remains to be elucidated. In these high-risk variants with increased arrhythmic risk, CMR offers additional information compared to the traditional diagnostic imaging. In addition to visualising regional wall-motion abnormalities, CMR can also identify reversible and irreversible 
myocardial damage (myocardial inflammation/oedema and myocardial scarring, respectively). CMR can also delineate the extent of LV dysfunction, LV outflow tract (LVOT) obstruction, any valvular dysfunction and LV thrombus. Some of these findings are directly linked to adverse prognosis.

In the current review, we describe the CMR findings in TCM, specifically focusing on the features that could be related to arrhythmic events and sudden death.

\section{ECG abnormalities and arrhythmias in TCM}

TCM can present with various acute electrocardiographic (ECG) changes, the commonest abnormality being STsegment elevation mimicking an ST-elevation myocardial infarction (STEMI) [4]. The time from symptom onset to presentation may account for the significant variability in the frequency of this finding in the published literature [5]. Usually, the ST-elevation is present in the precordial leads, but it may be seen in the inferior or lateral leads. The magnitude of ST-elevation is usually less prominent in TCM than in STEMI [5]; however, just a 12-lead ECG is insufficient for differentiating TCM from STEMI [6]. Other ECG changes commonly encountered include diffuse and often deep T-wave inversion and prolonged QTc interval. TCM can have four ECG phases: phase 1, initial STsegment elevation immediately after symptom onset; phase 2 , initial deep T-wave inversion after resolution of STsegment elevation from day 1 to 3 ; phase 3, transient improvement in $\mathrm{T}$-wave inversion in the subacute period; and phase 4, a second deeper T-wave inversion which may resolve early or persist beyond 1 year [7]. QTc interval varies according to time, and the most prolonged QTc interval may be observed in phase 2 [7, 8]. Malignant arrhythmias include torsades de pointes (TdP) caused by repolarisation abnormalities and QTc prolongation, which may be seen in up to $8 \%$ of cases [9]. Lethal arrhythmias in the acute phase (phase 1) are seen when the QTc is $>500 \mathrm{~ms}$ (Fig. 1). Generally, implantable cardioverter defibrillator (ICD) implantation should be considered in the presence of high-risk factors for long QT syndrome (QTc post-TCM $>500 \mathrm{~ms}$, prior syncope, previous cardiac arrest) [10].

In one meta-analysis, sudden cardiac death (SCD) after TCM has been reported in $1.1 \%$ during the index episode, with a further $0.5 \%$ of patients suffering a SCD weeks to months later [11].

The study primarily looked at the relationship between TCM and ventricular arrhythmias, reviewing a total 816 published cases. VF was reported in 15 reported cases (prevalence of $1.8 \%$ ). VT was reported in $18(2.2 \%)$, of which four $(0.5 \%)$ were specifically reported as sustained
VT, and eight $(1.1 \%)$ as non-sustained VT. One study with 88 patients $(15.6 \%$ of total) did not specify whether six cases of VT were sustained or non-sustained. They concluded that the combined case frequency with VF or sustained VT was $3.4 \%$ (28 of 816 cases).

Ventricular asystole had a prevalence of $0.6 \%$, often as a mode of presentation. Among these cases, there was only one describing an intermittent asystole developing from complete heart block.

Atrial fibrillation was reported in a cumulative 38 (4.7\% of 816 ) cases. Sinus node dysfunction was reported in $1.3 \%$ total cases.

Occasionally, malignant types of narrow complex tachycardia like fascicular ventricular tachycardia (VT) can occur in TCM, which may fail to respond to DC cardioversion (Fig. 2).

\section{Echocardiography and its limitation in TCM}

Among the clinical diagnostic criteria, left ventricular (LV) systolic dysfunction without evidence of coronary obstruction is the essential element for TCM diagnosis. The most common echocardiographic finding is the severe hypokinesia/akinesia of the mid-cavity and apical LV segments, giving the typical appearance of "apical ballooning". Although there is echocardiographic evidence of moderate-to-severe LV systolic dysfunction, these patients rarely experience acute heart failure symptoms. Possible explanations include the transitory nature of the condition, preservation of the LV stroke volume due to acute ballooning (dilatation) of the apical segments or perhaps due to compensatory hyper-contractility of the unaffected basal myocardial segments. The mechanisms underlying the cardiac dysfunction in TCM are poorly understood and have not been extensively investigated. Possible factors contributing to this unique apical localisation of TCM is the anatomical structure of the apex without a three-layered myocardial structure (as in the basal and mid-cavity segments); an easy loss of LV apex elasticity after excessive expansion; LV apex as the border zone (locus minoris resistentiae) of the perfusion area of major coronary arteries; a relative delay of functional recovery from global dysfunction [4].

A recent study by Medeiros et al. [12] compared systolic and diastolic mechanics of TCM compared with left anterior descending artery (LAD) MI and normal controls. LV volumes, diastolic pressures and diastolic stiffness were higher in TCM and LAD MI patients than in control subjects but no different from each other. Moreover, in the TCM group, EF improved from presentation to follow-up from $34.7 \pm 10.5$ to $60.7 \pm 7.4 \%$ and with a reduction in $\mathrm{E} / \mathrm{E}^{\prime}$ ratio. These data demonstrated that not only TCM is 


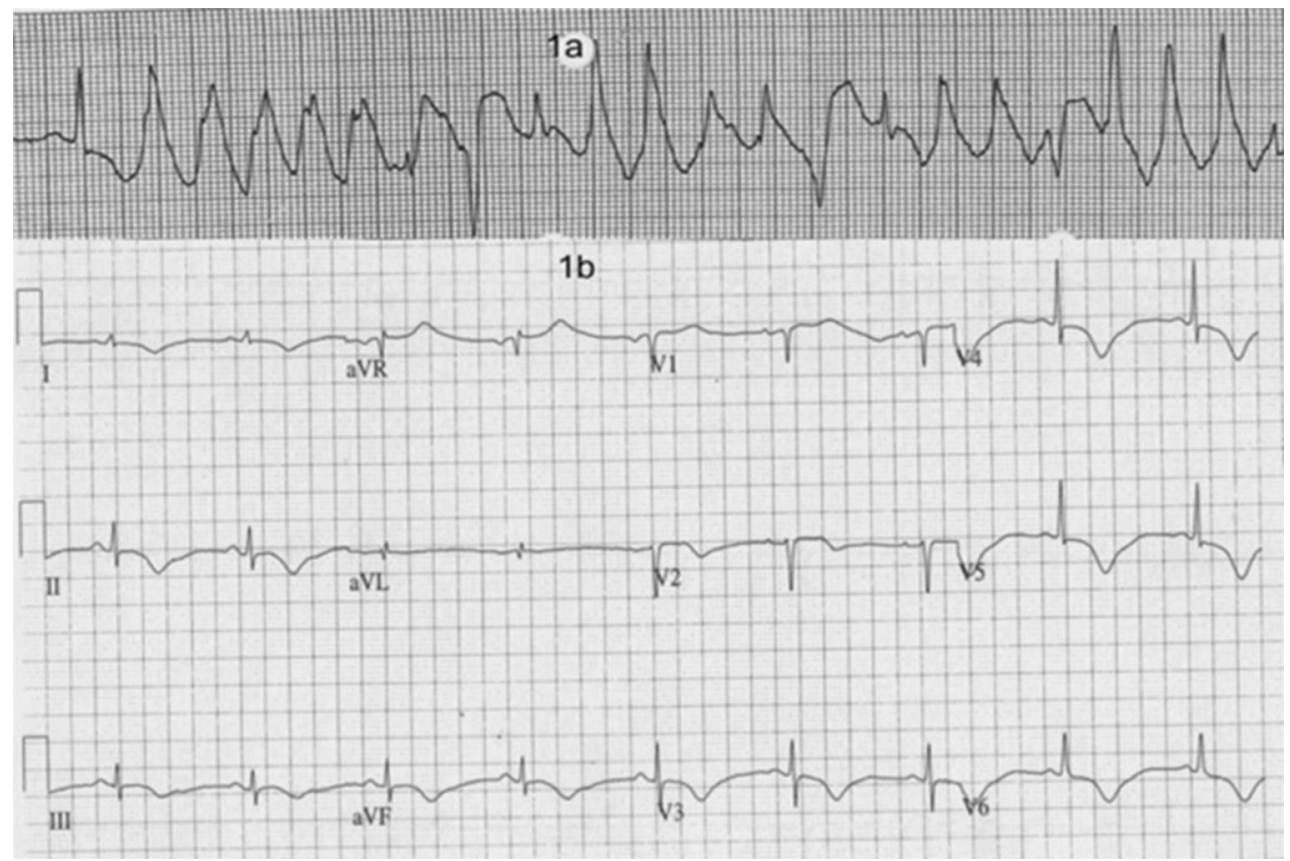

Fig. 1 a Defibrillator monitor showing polymorphic VT and b 12 lead ECG showing sinus rhythm with markedly prolonged QTc


Fig. 2 a TCM presenting with narrow complex tachycardia (likely fascicular VT), and b Defibrillator monitor showing lack of response to DC cardioversion

characterised by systolic dysfunction, but LV filling pressure is also increased suggesting LV diastolic impairment. These findings have been recently confirmed by a CMR study showing that diastolic dysfunction, evaluated by peak filling rate and atrial measurements, is persistently impaired in TCM even after systolic recovery [13]. Longitudinal strain and strain rate have also been recently investigated in TCM, demonstrating impairment of both parameters not only during the acute phase but persisting even during follow-up [14, 15]. Echocardiography can sometimes present limitations in the diagnosis of TCM given its intrinsic limitation in fully visualising the apex, and limited detection of the endocardial border from apical trabeculations [16]. This could result in either the overestimation of LV systolic function and stroke volume or underestimation of apical dysfunction. 


\section{CMR findings in TCM}

The characteristic mid-cavity to apical regional wall-motion abnormalities ("apical ballooning") with sparing of the LV basal segments can be picked up by LV angiogram as well as transthoracic echocardiography. The added diagnostic value of CMR is in its (1) high spatial resolution, 3D image acquisition and (2) non-invasive myocardial tissue characterisation.

The former results in a clear detection of cardiac chambers and endocardial contours, whilst the latter allows the detection of myocardial oedema (markedly present in TCM) and myocardial scarring (absent in TCM). The latter represents indeed the main differential diagnostic criteria between TCM and AMI

CMR can also provide additional information on the presence of LV apical thrombus, valvular abnormalities and LVOT obstruction. Some of these findings are directly linked to adverse prognosis.

Typically, T2-weighted sequences are used to image myocardial oedema, and it commonly identifies the presence of circumferential oedema of the apical to mid-cavity myocardium (Fig. 3a) matching with the regional wallmotion abnormalities [17]. As myocardial oedema is a transient dynamic phenomenon (normally progressively resolving between 3 and 6 months), it is advisable that the scans need to be carried out within the first few weeks from the acute presentation. The myocardial oedema dissipates without any myocardial scarring and with complete functional recovery [17]. A few studies have shown the presence of subtle late gadolinium enhancement in TCM [18], but experimental data show that delayed washout of gadolinium may be caused by increased interstitial water content such that associated with transient myocardial oedema, and not just by irreversible myocardial necrosis/fibrosis as observed in AMI [19]. The subtle changes are called myocardial oedema-related late myocardial enhancement, and they do not represent true scarring. The distribution of myocardial oedema matching the wall-motion abnormalities with no or subtle delayed enhancement helps in distinguishing TCM from MI and myocarditis. The exact pathophysiological mechanisms underlying the development of myocardial oedema in TCM remain unclear, but inflammation, increased LV wall stress and/or transient ischaemia (myocardial stunning) appear pivotal.

The clinical spectrum of TCM is considerably broad and a prospective study evaluating a precise CMR pattern related to outcome is lacking. The largest multi-centre study in this field enrolling 239 consecutive patients, reported a moderate-to-severe LV function reduction in all patients associated with myocardial oedema in $81 \%$ of patients with a distinct transmural, mid-ventricular to apical regional distribution pattern matching the distribution of $\mathrm{LV}$ dysfunction. On contrary, focal or patchy LGE was detected in $9 \%$ of enrolled subjects. In addition, one-third of patients demonstrated pleural and pericardial effusion particularly those with biventricular ballooning and evidence of myocardial inflammation [20]. During follow-up, four patients died. Another study showed that LGE in the acute phase was associated with acute cardiogenic shock as well as with disease severity and prolonged recovery. However, the signal intensity of LGE was lower than that
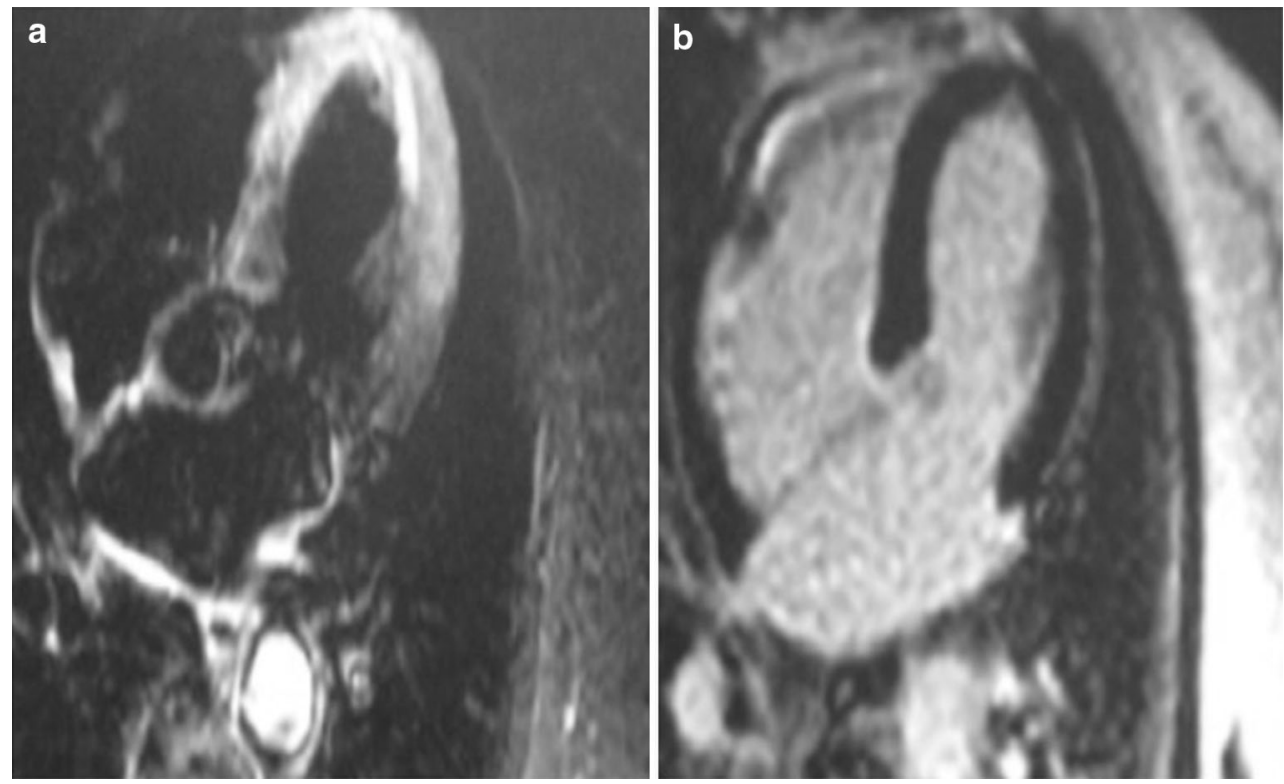

Fig. 3 a Four-chamber view showing mid-apical oedema in T2-weighted sequences and $\mathbf{b}$ no evidence of significant late gadolinium enhancement in T1-weighted inversion recovery post-contrast sequences 
usually documented in cases of MI or myocarditis [21]. Whilst initially it was believed that lack of LGE was a necessary condition to diagnose TCM and would thus help differentiate it from MI, in the study by Haghi et al. [22], $88 \%$ of patients showed a patchy type of LGE. In view of these findings, Rolf et al. [23] performed multiple endomyocardial biopsies in sites with and without LGE showing a significantly higher extracellular matrix containing collagen-1 in LGE areas. In another study, Neil et al. [24] found increased T2-weighted signal intensity particularly at the apical cavity in all TCM patients that persisted after 3 months and was inversely related to myocardial strain. In accordance with the above-mentioned data, myocardial oedema on CMR is the only recognised feature in TCM, reflecting acute inflammation and myocardial injury [25]. Nakamori et al. [26] monitored 23 TCM patients. Upon presentation, all subjects experienced wall-motion abnormalities from the mid-ventricular to apical regions of the LV. Myocardial oedema on T2weighted magnetic resonance imaging was observed in $96 \%$, and late gadolinium enhancement was observed in $22 \%$. All instances of LGE were observed in an area that exhibited both wall-motion abnormalities and oedema in a transmural distribution. In TCM, the contrast-to-noise ratio was significantly lower than that of infarcted segments in patients with acute myocardial infarction.

All these data suggest a role of active inflammation, although the precise contribution of myocardial inflammation for LV dysfunction in TCM is still unclear. It remains to be established whether inflammation is a direct cause of the syndrome or a secondary phenomenon due to sympathetic overdrive and/or microvascular ischaemia. The combination of typical regional wall-motion abnormalities, the presence of reversible myocardial injury and the absence of significant irreversible tissue injury are all potential diagnostic criteria, but the evidence of specific finding related to outcome should be prospectively tested. In the absence of studies that specifically report a clear CMR criteria classification, the diagnosis continues to be entirely case dependent and should be individualised in accordance with the patient's characteristics, angiographic findings and hemodynamic requirements at the time of presentation.

\section{Correlating the ECG with CMR}

T-wave inversion and QT interval prolongation in typical TCM are caused by an intracardiac gradient (apicobasal) of myocardial oedema which, in turn, gives rise to regional dispersion of action potential durations [27]. Furushima et al. [27] measured the intracardiac repolarisation gradients between the apical and basal sites of the LV in a patient with typical TCM and negative T-waves/QT interval prolongation in leads II, III, aVF and V2-V6 and demonstrated that repolarisation times increased from the basal to the apical sites in both the epicardium and the endocardium. This observation confirms the hypothesis that inverted T-waves seen in TCM are the result of LV dispersion of repolarisation caused by abnormal prolongation of action potentials in the affected myocardial regions. A study by Perazzolo Marra et al. [17] looked at the ECG and CMR findings in 20 consecutive TCM patients. A linear correlation of the apical-basal ratio of T2-weighted signal intensity with ECG repolarisation indices such as negative T-wave magnitude, sum of the amplitudes of negative T-waves and maximum corrected QT interval has been shown. Interestingly, the repolarisation changes were unrelated to either late gadolinium enhancement or quantitative cine parameters.

Other malignant arrhythmias like fascicular VT can also be occasionally encountered in TCM. Increased automaticity or increased catecholaminergic drive with related cytosolic calcium overload rather than a re-entry is the pathophysiologic cause. Hence, the lack of scar in CMR can potentially explain the mechanism of this unique arrhythmia.

Future experimental studies are needed to elucidate the cellular mechanisms responsible for oedema-related action potential changes.

\section{Conclusion}

Although TCM is usually considered to be a benign reversible condition, its arrhythmic risk is increasingly recognised. CMR is a promising new diagnostic tool that not only confirms the diagnosis but also help to risk stratify the patients with potential higher arrhythmic risk. Further prospective studies are warranted to demonstrate this hypothesis in a larger scale patients group.

Acknowledgments This work was supported by the Bristol NIHR Cardiovascular Biomedical Research Unit at the Bristol Heart Institute. The views expressed are those of the authors and not necessarily those of the National Health Service, National Institute for Health Research, or Department of Health.

Conflict of interest None.

\section{References}

1. Prasad A, Lerman A, Rihal CS (2008) Apical ballooning syndrome (Tako-Tsubo or stress cardiomyopathy): a mimic of acute myocardial infarction. Am Heart J [Internet]. (cited 2013 Oct 25);155(3):408-17. http://www.ncbi.nlm.nih.gov/pubmed/18294473

2. Kawai S, Kitabatake A, Tomoike H (2007) Guidelines for diagnosis of takotsubo (ampulla) cardiomyopathy. Circ J [Internet]. 
(cited 2013 Oct 26);71(6):990-2. http://www.ncbi.nlm.nih.gov/ pubmed/17527002

3. Wittstein IS, Thiemann DR, Lima JAC, Baughman KL, Schulman SP, Gerstenblith G, et al. (2005) Neurohumoral features of myocardial stunning due to sudden emotional stress. N Engl $\mathbf{J}$ Med [Internet]. [cited 2013 Oct 19];352(6):539-48. http://www. ncbi.nlm.nih.gov/pubmed/15703419

4. Tsuchihashi K, Ueshima K, Uchida T, Oh-mura N, Kimura K, Owa M, et al. (2001) Transient left ventricular apical ballooning without coronary artery stenosis: a novel heart syndrome mimicking acute myocardial infarction. Angina pectoris-myocardial infarction investigations in Japan. J Am Coll Cardiol [Internet]. (cited 2013 Oct 26);38(1):11-8. http://www.ncbi.nlm.nih.gov/ pubmed/11451258

5. Bybee KA, Kara T, Prasad A, Lerman A, Barsness GW, Wright RS, et al. (2004) Systematic review: transient left ventricular apical ballooning: a syndrome that mimics ST-segment elevation myocardial infarction. Ann Intern Med [Internet]. (cited 2013 Oct 27);141(11):858-65. http://www.ncbi.nlm.nih.gov/pubmed/ 15583228

6. Bybee KA, Motiei A, Syed IS, Kara T, Prasad A, Lennon RJ, et al. (2007) Electrocardiography cannot reliably differentiate transient left ventricular apical ballooning syndrome from anterior ST-segment elevation myocardial infarction. J Electrocardiol [Internet]. (cited 2013 Oct 27);40(1):38.e1-6. http://www.ncbi. nlm.nih.gov/pubmed/17067626

7. Dastidar AG, Frontera A, Petersen M. (2014) A case of malignant arrhythmia in Takotsubo Cardiomyopathy. J Electrocardiol [Internet]. Elsevier (cited 2014 Mar 26). http://www.jecgonline.com/ article/S0022-0736(14)00074-0/abstract

8. Kurisu S, Inoue I, Kawagoe T, Ishihara M, Shimatani Y, Nakamura S, et al. (2004) Time course of electrocardiographic changes in patients with tako-tsubo syndrome: comparison with acute myocardial infarction with minimal enzymatic release. Circ J [Internet]. (cited 2013 Oct 27);68(1):77-81. http://www.ncbi.nlm. nih.gov/pubmed/14695470

9. Madias C, Fitzgibbons TP, Alsheikh-Ali AA, Bouchard JL, Kalsmith B, Garlitski AC, et al. (2011) Acquired long QT syndrome from stress cardiomyopathy is associated with ventricular arrhythmias and torsades de pointes. Heart Rhythm [Internet]. (cited 2013 Oct 30);8(4):555-61. http://www.ncbi.nlm.nih.gov/ pubmed/21146632

10. Rotondi F, Manganelli F. (2013) Takotsubo cardiomyopathy and arrhythmic risk: the dark side of the moon. Eur Rev Med Pharmacol Sci [Internet]. (cited 2013 Oct 30);17(1):105-11. http:// www.ncbi.nlm.nih.gov/pubmed/23329530

11. Syed FF, Asirvatham SJ, Francis J (2011) Arrhythmia occurrence with takotsubo cardiomyopathy: a literature review. Europace [Internet]. (cited 2013 Oct 30);13(6):780-8. http://europace. oxfordjournals.org/content/13/6/780

12. Medeiros K, O'Connor MJ, Baicu CF, Fitzgibbons TP, Shaw P, Tighe DA, et al. (2014) Systolic and diastolic mechanics in stress cardiomyopathy. Circulation [Internet]. (cited 2014 Oct 11); 129(16):1659-67. http://www.ncbi.nlm.nih.gov/pubmed/24503950

13. Ahtarovski KA, Iversen KK, Christensen TE, Andersson H, Grande P, Holmvang L, et al. (2014) Takotsubo cardiomyopathy, a two-stage recovery of left ventricular systolic and diastolic function as determined by cardiac magnetic resonance imaging. Eur Heart J Cardiovasc Imaging [Internet]. (cited 2014 Oct 11);15(8):855-62. http://www.ncbi.nlm.nih.gov/pubmed/2452 5137

14. Loiske K, Waldenborg M, Fröbert O, Rask P, Emilsson K. (2011) Left and right ventricular systolic long-axis function and diastolic function in patients with takotsubo cardiomyopathy. Clin Physiol Funct Imaging [Internet]. (cited 2014 Oct 11);31(3):203-8. http:// www.ncbi.nlm.nih.gov/pubmed/21138516
15. Heggemann F, Weiss C, Hamm K, Kaden J, Süselbeck T, Papavassiliu T, et al. (2009) Global and regional myocardial function quantification by two-dimensional strain in Takotsubo cardiomyopathy. Eur J Echocardiogr [Internet]. (cited 2014 Oct 11);10(6): 760-4. http://www.ncbi.nlm.nih.gov/pubmed/19477932

16. Cisneros S, Duarte R, Fernandez-Perez GC, Castellon D, Calatayud J, Lecumberri I, et al. (2011) Left ventricular apical diseases. Insights Imaging [Internet]. (cited 2014 Oct 11);2(4): 471-82. http://www.pubmedcentral.nih.gov/articlerender.fcgi?ar tid $=3259385 \&$ tool $=$ pmcentre $z \&$ rendertype $=$ abstract

17. Perazzolo Marra M, Zorzi A, Corbetti F, De Lazzari M, Migliore F, Tona F, et al. (2013) Apicobasal gradient of left ventricular myocardial edema underlies transient $\mathrm{T}$-wave inversion and QT interval prolongation (Wellens' ECG pattern) in Tako-Tsubo cardiomyopathy. Heart Rhythm [Internet]. (cited 2013 Oct 27);10(1):70-7. http://www.ncbi.nlm.nih.gov/pubmed/ 22975421

18. Avegliano G, Huguet M, Costabel JP, Ronderos R, Bijnens B, Kuschnir P, et al. (2011) Morphologic pattern of late gadolinium enhancement in Takotsubo cardiomyopathy detected by early cardiovascular magnetic resonance. Clin Cardiol [Internet]. (cited 2013 Oct 30);34(3):178-82. http://www.ncbi.nlm.nih.gov/pubmed/ 21400545

19. Inoue S, Murakami Y, Ochiai K, Kitamura J, Ishibashi Y, Kawamitsu H, et al. (1999) The contributory role of interstitial water in Gd-DTPA-enhanced MRI in myocardial infarction. J Magn Reson Imaging [Internet]. [cited 2013 Oct 30];9(2): 215-9. http://www.ncbi.nlm.nih.gov/pubmed/10077016

20. Eitel I, von Knobelsdorff-Brenkenhoff F, Bernhardt P, Carbone I, Muellerleile K, Aldrovandi A, et al. (2011) Clinical characteristics and cardiovascular magnetic resonance findings in stress (takotsubo) cardiomyopathy. JAMA [Internet]. (cited 2014 Oct 11);306(3):277-86. http://www.ncbi.nlm.nih.gov/pubmed/ 21771988

21. Naruse Y, Sato A, Kasahara K, Makino K, Sano M, Takeuchi Y, et al. (2011) The clinical impact of late gadolinium enhancement in Takotsubo cardiomyopathy: serial analysis of cardiovascular magnetic resonance images. J Cardiovasc Magn Reson [Internet]. (cited 2014 Oct 11);13(1):67. http://www.jcmr-online.com/con tent/13/1/67

22. Haghi D, Fluechter S, Suselbeck T, Kaden JJ, Borggrefe M, Papavassiliu T. (2007) Cardiovascular magnetic resonance findings in typical versus atypical forms of the acute apical ballooning syndrome (Takotsubo cardiomyopathy). Int J Cardiol [Internet]. Elsevier (cited 2014 Oct 11];120(2):205-11. http:// www.internationaljournalofcardiology.com/article/S0167527306 013465/fulltext

23. Rolf A, Nef HM, Möllmann H, Troidl C, Voss S, Conradi G, et al. (2009) Immunohistological basis of the late gadolinium enhancement phenomenon in tako-tsubo cardiomyopathy. Eur Heart J [Internet]. (cited 2014 Oct 11);30(13):1635-42. http:// www.ncbi.nlm.nih.gov/pubmed/19389788

24. Neil C, Nguyen TH, Kucia A, Crouch B, Sverdlov A, Chirkov Y, et al. (2012) Slowly resolving global myocardial inflammation/ oedema in Tako-Tsubo cardiomyopathy: evidence from T2weighted cardiac MRI. Heart [Internet]. (cited 2014 Oct 11);98(17):1278-84. http://www.ncbi.nlm.nih.gov/pubmed/2279 1656

25. Iacucci I, Carbone I, Cannavale G, Conti B, Iampieri I, Rosati R, et al. (2013) Myocardial oedema as the sole marker of acute injury in Takotsubo cardiomyopathy: a cardiovascular magnetic resonance (CMR) study. Radiol Med [Internet]. (cited 2014 Oct 11);118(8):1309-23. http://www.ncbi.nlm.nih.gov/pubmed/2371 6287

26. Nakamori S, Matsuoka K, Onishi K, Kurita T, Ichikawa Y, Nakajima H, et al. (2012) Prevalence and signal characteristics of 
late gadolinium enhancement on contrast-enhanced magnetic resonance imaging in patients with takotsubo cardiomyopathy. Circ J [Internet] (cited 2014 Oct 11);76(4):914-21. http://www. ncbi.nlm.nih.gov/pubmed/22293447
27. Furushima H, Chinushi M, Sanada A, Aizawa Y. (2008) Ventricular repolarization gradients in a patient with takotsubo cardiomyopathy. Europace [Internet]. (cited 2013 Oct 30);10(9): 1112-5. http://www.ncbi.nlm.nih.gov/pubmed/18567569 